\title{
RESEARCH ON SIMULATION OF BANKING SYSTEM FOR MEASURING THE PRODUCTIVITY OF THE RESOURCES INVOLVED WITHIN THE SYSTEM
}

\author{
Sarang A Katkar ${ }^{1}$, Ronak Modh ${ }^{2}$, Sunny Bhavan Sall ${ }^{3}$, Sanjay Kulkarni ${ }^{4}$ \\ ${ }^{1}$ Industrial Engineering, bvbcet, Hubli. katkar_sarang@rediffmail.com \\ ${ }^{2}$ Software Developer, Qunniox Consultancy Services, Mumbai.ronak.r.modh@gmail.com \\ ${ }^{3}$ Principal, Sardar Vallabhai Patel Polytecnic, Hubli.sunny_sall@yahoo.co.in \\ ${ }^{4}$ Prof Industrial Engineering, bvbcet, Hubli. sanjaykulkarni@bvb.edu
}

\begin{abstract}
The Simulation of banking system is mainly focused as to how much average time each type of customer takes in a bank for a transaction and how much percentage of time the teller is busy. The Arena software plays a vital role in determining these aspects nevertheless it can help us in decision making by giving a probable forecast as to how we might expect for example-the number of products that can be sold if the process, our current sales, methods go on the same way. This paper will serve as reference as in what ways we could alter the very way of the system to make it more efficient and excluding anything which might bottleneck the full performance. The paper is concluded with suggestions on improvement in the banking process.
\end{abstract}

Keywords: entity, resources, utilization, idle time.

\section{INTRODUCTION}

In a banking sector, the key motive is to serve the customer effectively while maintaining their balance by extracting profits through interests and many other ways this is usually achieved with the help of efficient and effective use of methods and employees.

This simulation which helps to presage the future will help not only to know the inefficient aspects of a system but also help to predict the future condition of the organisation by giving prescient profit or loss of a company if its current way of carrying out the tasks goes on the same way.

The simulation will help the banking system in by pointing out the areas where there is idle time, or lack of efficiency in there system and consequently it will helps us to decide as to how we should proceed to improve the system to work better and gain profit. For example if an employee in a bank is not productive or is not needed or maybe he is not being used to a proper extent we can fire him, which in turn will act as profit to the organisation as his salary is saved.

\section{METHODOLOGY}

Work plan is the systematic, theoretical analysis of the methods applied to a field study, or the theoretical analysis of the body of methods and principles as associated with a branch of knowledge.

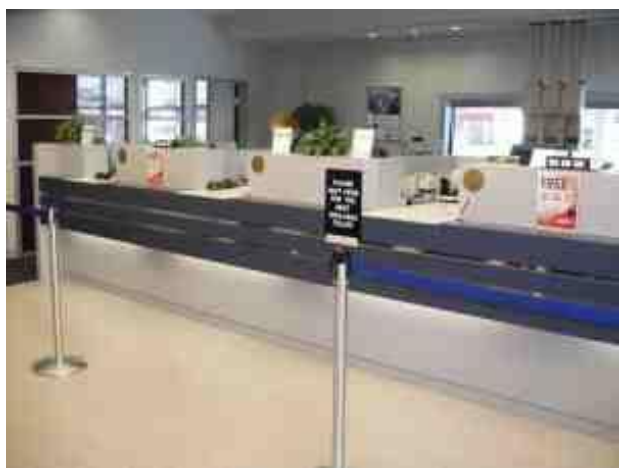

Figure 1: Teller/transaction section

Arena uses the "siman" processor and simulation language.Statistical data such as cycle time and work in progress levels can be recorded and outputted as reports.

In Arena, the user builds an experiment model by placing modules (boxes of different shapes) that represent processes or logic. Connector lines are used to join these modules together and to specify the flow of entities. While modules have specific actions relative to entities, flow, and timing, the precise representation of each module and entity relative to real-life objects is subject to the modeler.

Statistical data such as cycle time and work in progress levels can be recorded and outputted as reports.

- Evaluate potential alternatives to determine the best approach to optimizing performance.

- Understand system performance based on key metrics such as costs, throughput, cycle times, equipment utilization and resource availability.

- $\quad$ Reduce risk through rigorous simulation and testing of 
process changes before committing significant capital or resource expenditures.

- Determine the impact of uncertainty and variability on system performance.

- Run "what-if" scenarios to evaluate proposed process changes.

\subsection{Process Flow Analysis}

The flow analysis method offers such a means of considering essential ways and the level at which the problem is to be addressed. The flow of system to be designed must satisfy the requirements enlisted., no matter what physical components might be used. The problem level is decided by establishing a boundary around coherent subset of functions.

\section{Flow analysis}

- General accounts customer should be assigned to either respective GE teller 1 and 2 .

- Business accounts teller should be assigned to respective BA teller 3 and 4 .
- General accounts transaction takes 6+/-5 mins.

- Business accounts transaction 15+/-10 mins.

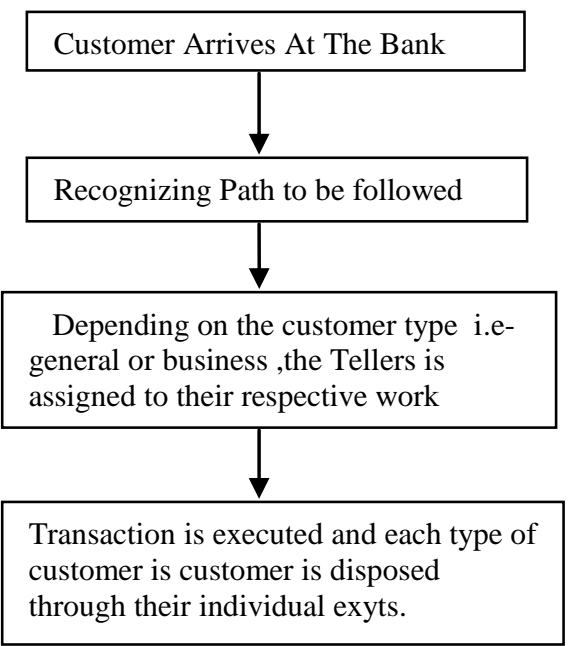

Figure 2: Flow diagram of process

\subsection{Design}

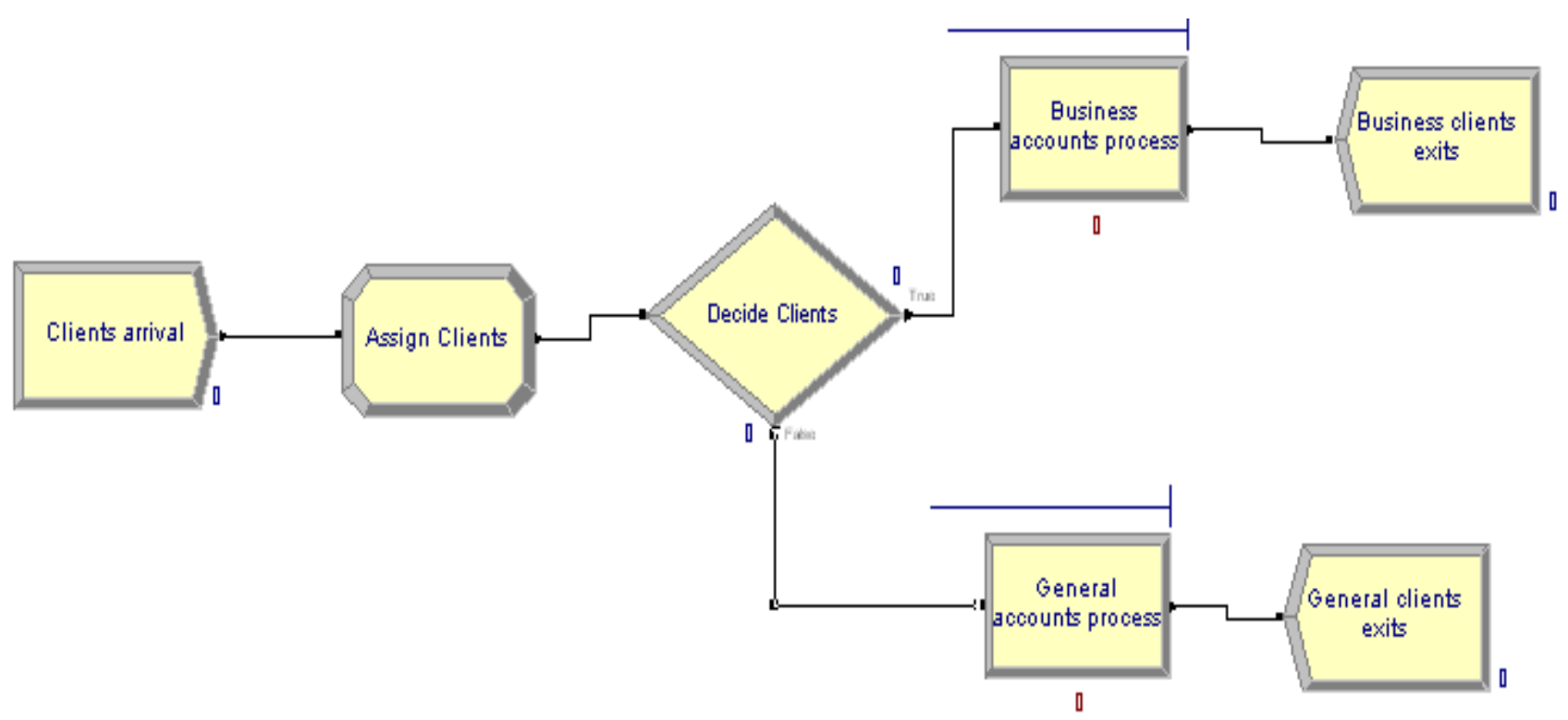

Figure 3: Final Flow

The above is the final flow of the bank.Here the clients arrive at the bank at a rate of every $3+/-1$ mins.Of the the clients $33 \%$ are business accounts. Clients randomly choose between the two tellers for each account type.

The processing time required for General accounts transaction is $6+/-5$ mins.

And for Business accounts transaction 15+/-10 mins.
The entities involved are customer or client, the tellers, the resources are the two types of account - there utilization is of utmost importance

The above, has been simulated for 50 transactions involving both the accounts. 


\subsection{Detailed Results and Analysis}

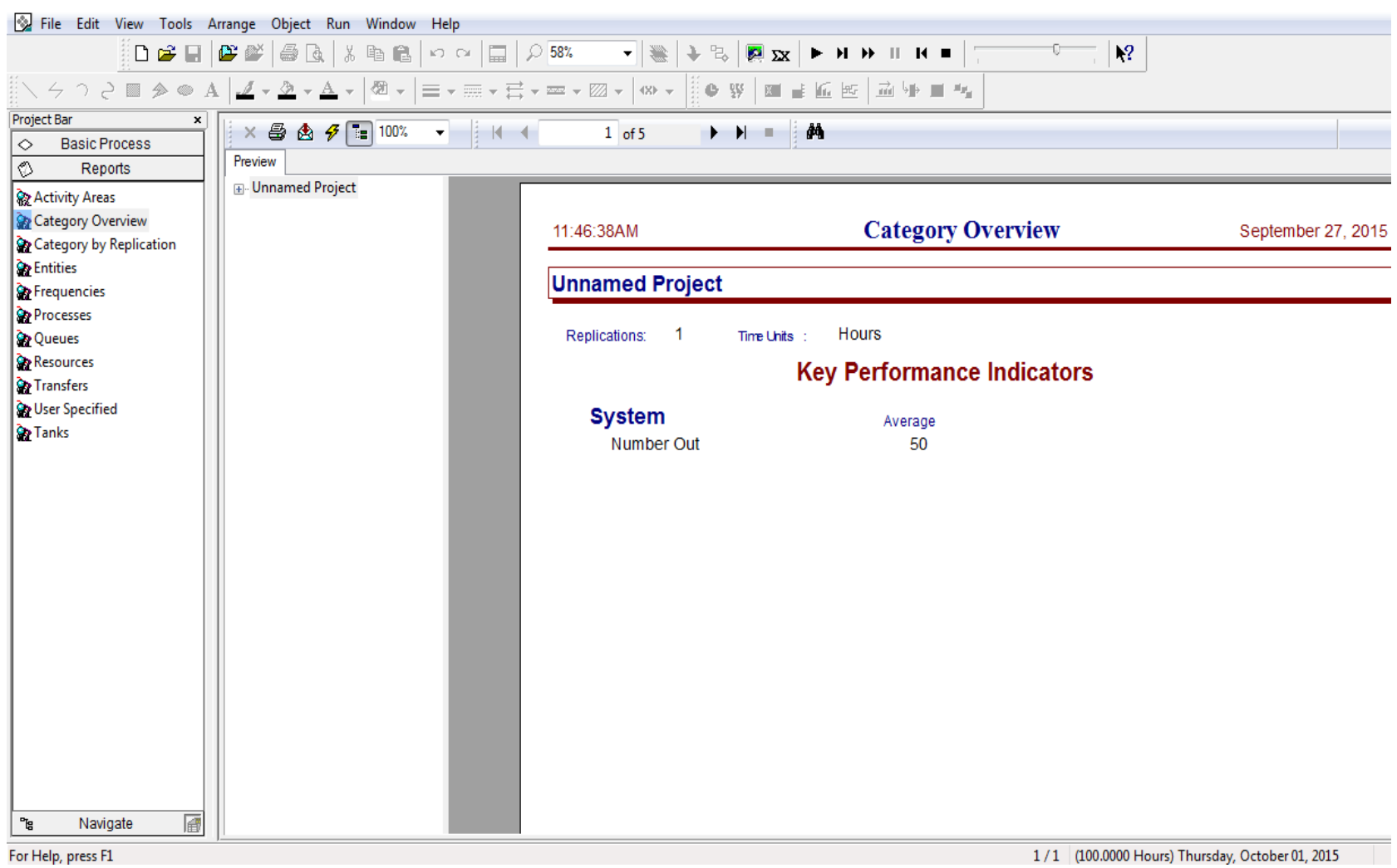

Figure 4: Number out

As given, the average number out -entities i.e.- customers is 50 as specified which represent 50 transactions taking place and a replication length is 1 .

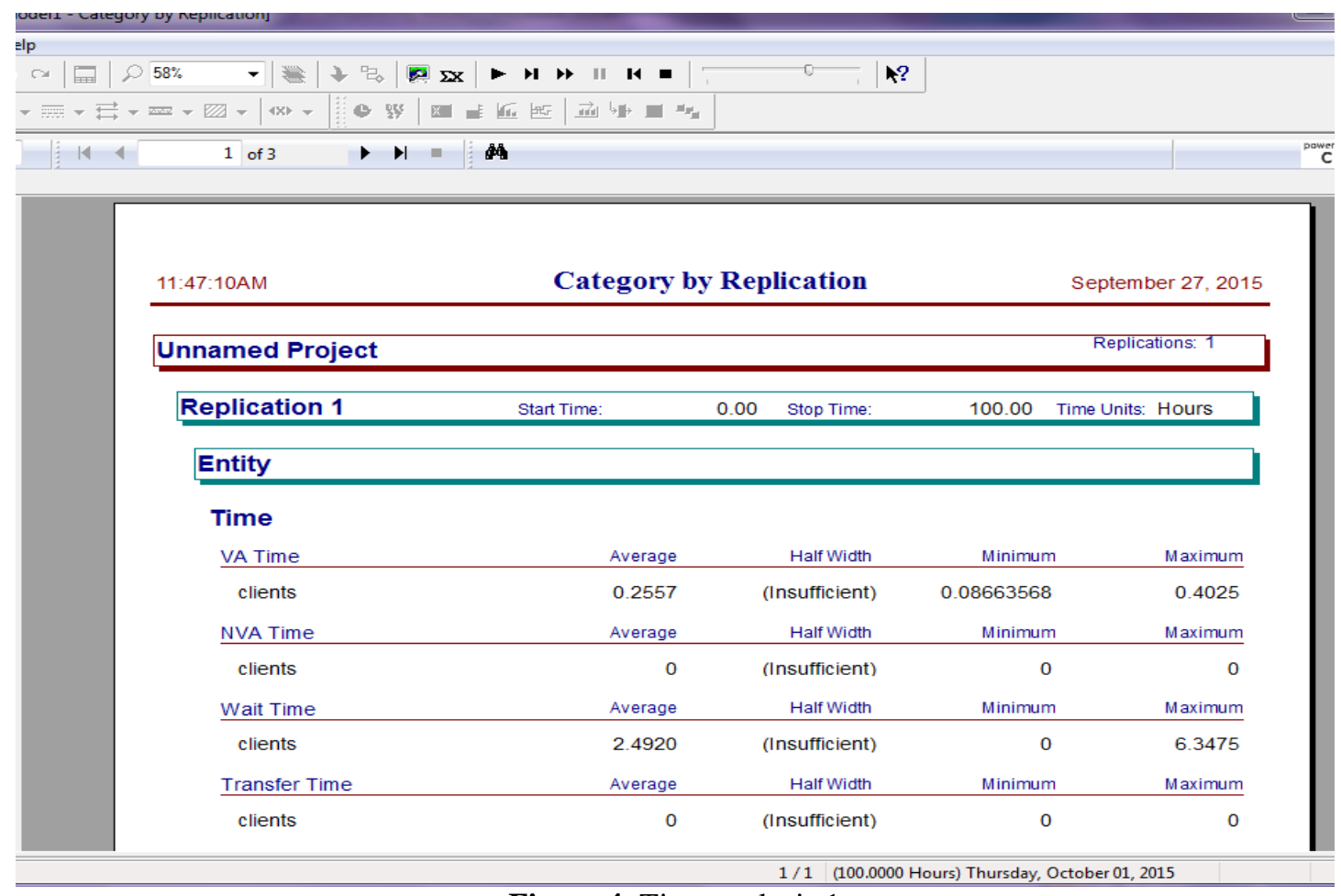

Figure 4: Time analysis.1 
This above stats show that the client or customer takes average 2.4920 and a maximum of 6.3475 wait time as shown above.

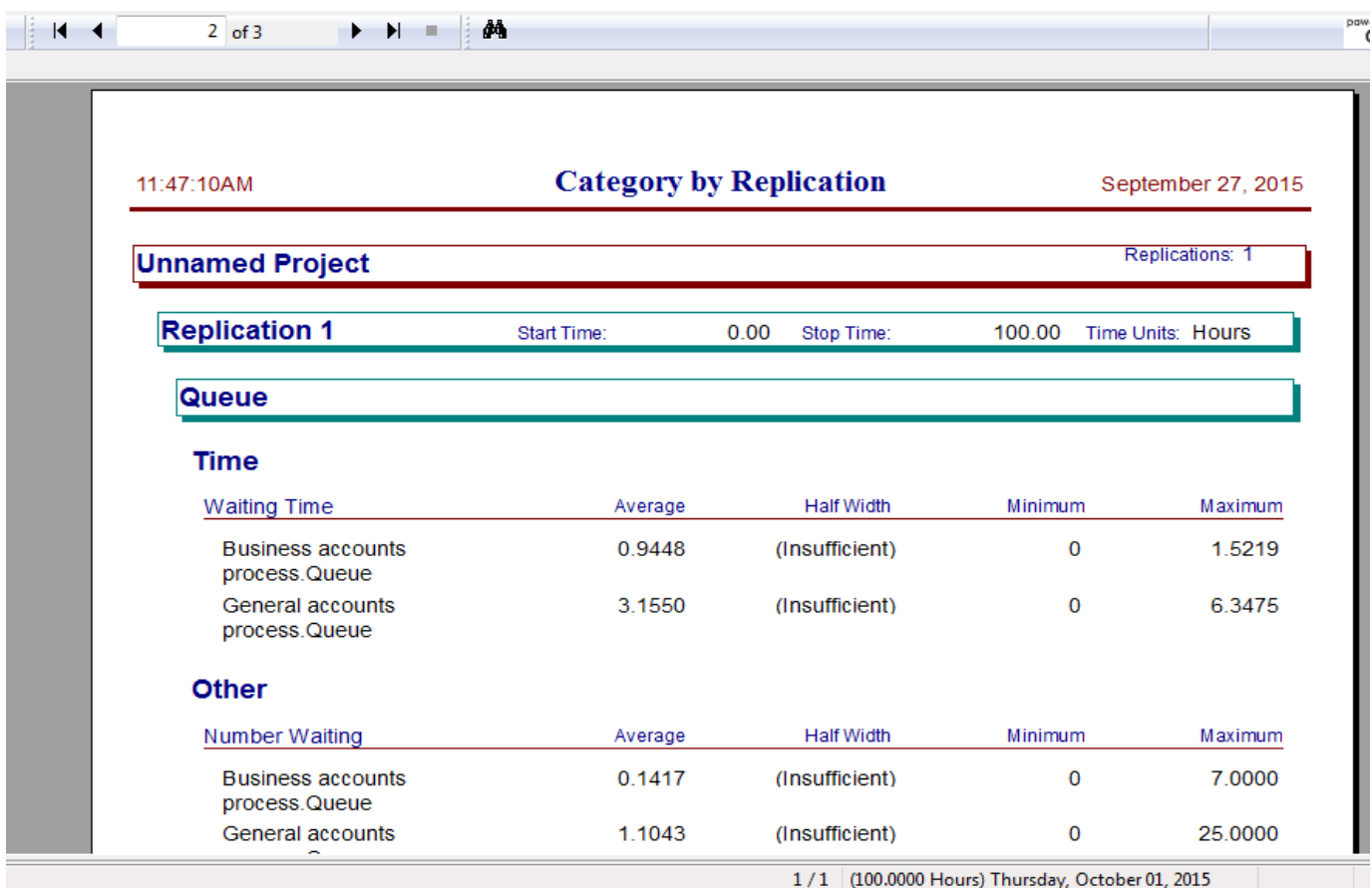

Figure 5: Queue time analysis

According to the data, the business accounts process queue takes lesser time compare to the general account queue that implies that the general accounts should or might probably increase their employees which nevertheless is contingent upon the utilization of that particular account.

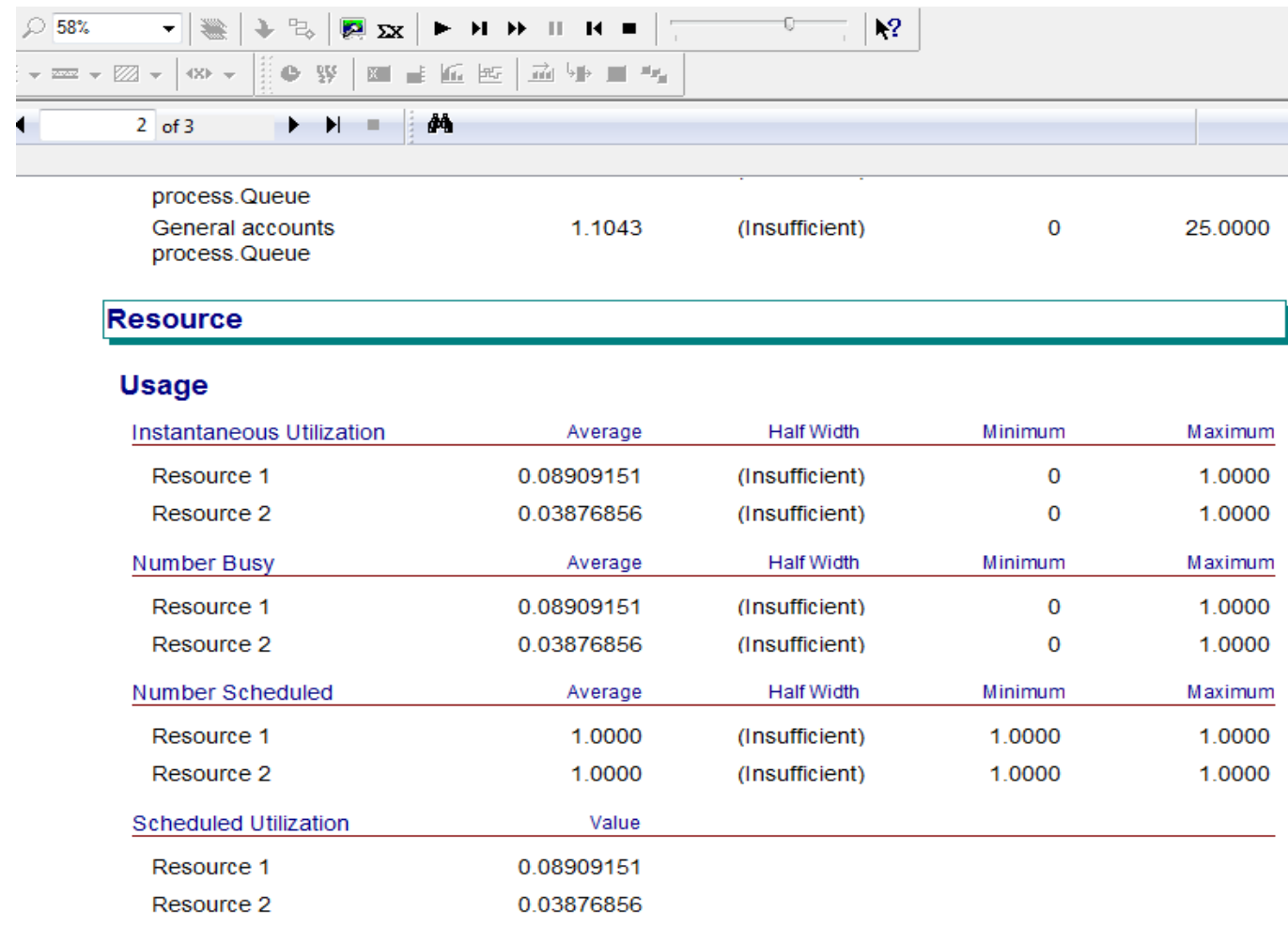


Figure 6: Utilization analysis

It can inferred from the data above that the resource 1 is utilized relatively more than than resource 2 which is the utilized more that means that the two tellers are being general account type.

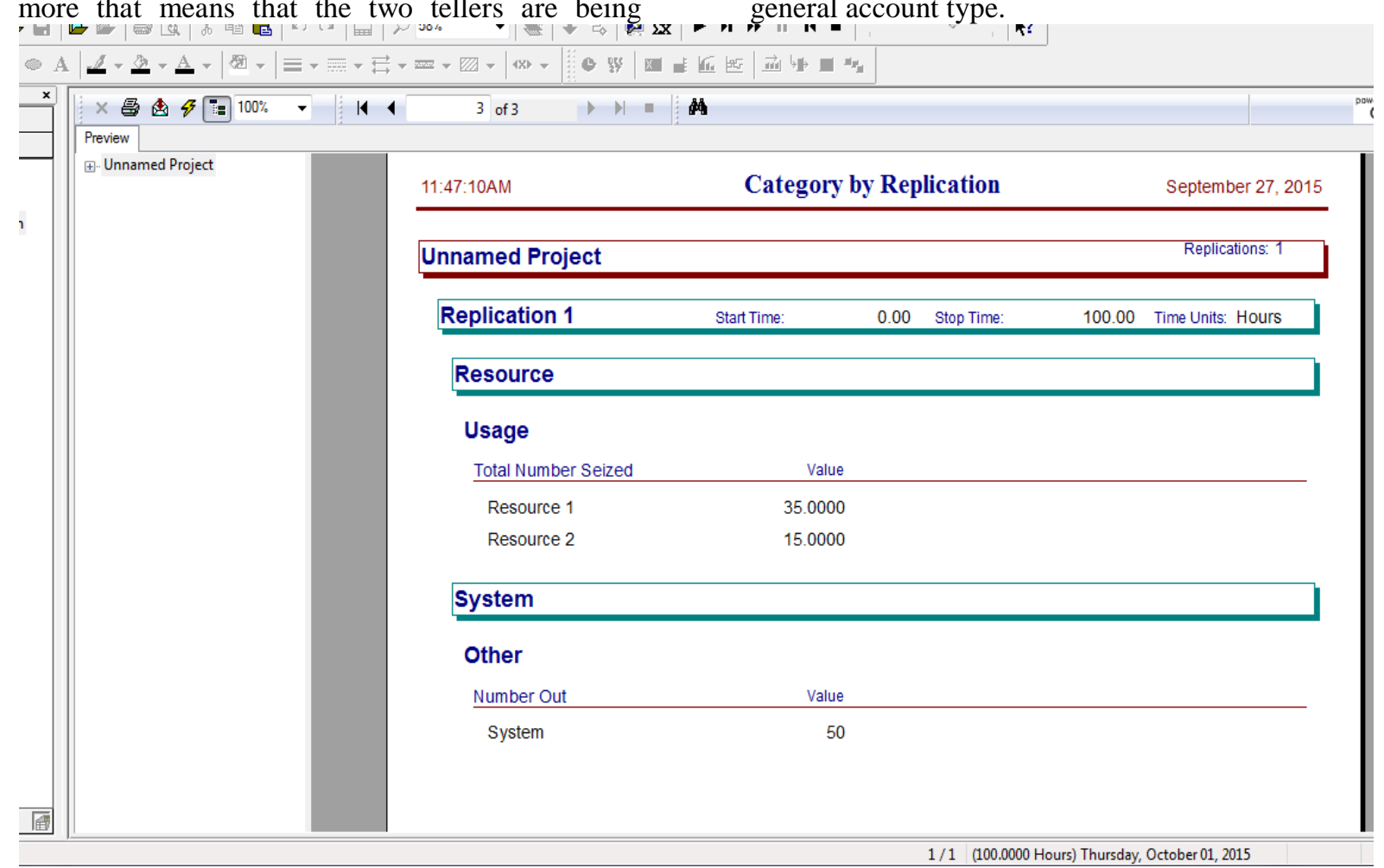

Figure 7: Resource output

The total number of clients processed in resource 1 is 35

whereas in resource 2 is 15 . 


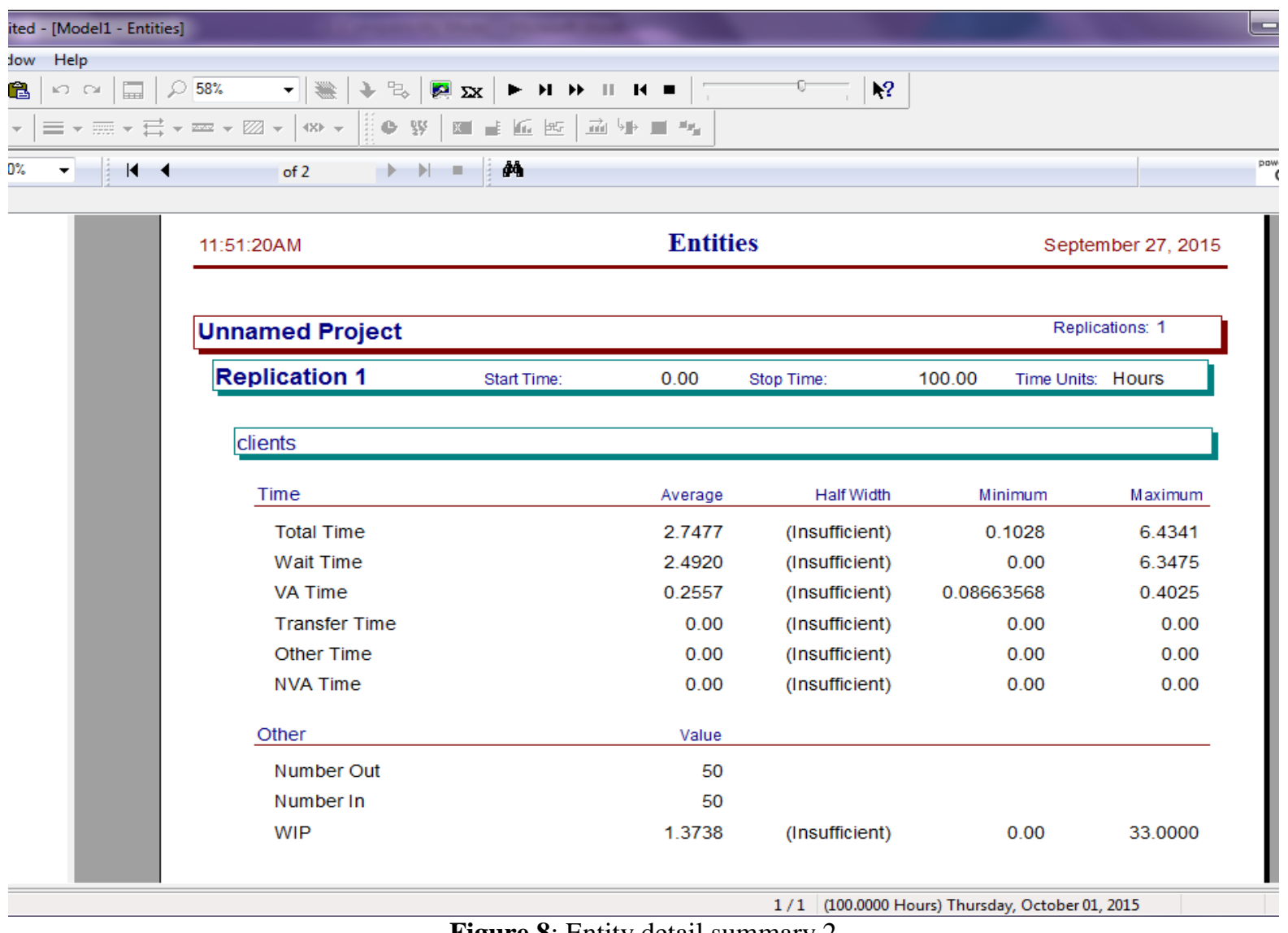

Figure 8: Entity detail summary.2

The above data, enlisting all the required times.

\section{CONCLUSIONS}

It can be inferred from the above results and its analysis that the waiting time and WIP is more for general accounts type because of more number of entities entering in that type nevertheless the tellers or employees are not being used or utilized effectively for desired performance however, the business type has higher utilization and relatively lower WIP and waiting time and hence it turns out to be efficient and productive.

The general accounts type utilization can be improved by including more tellers or reducing 1 teller so that the utilization of it is maximum however there is a drawback to this process that it increases waiting time. To solve both issues of waiting time and utilization of resources we could remove that entity which has more idle time or aggrandize the number of employees for that type.

\section{REFERENCES}

[1] E. Zimmers, "Introduction to Simulation"

[2] Mikkel P. Groover, "Simulation methodology "

[3] Shekar K.C, "Banking theory and practice"

[4] M. Groover, "Arena software simulation techniques"

[5] Zeigler, "Theory on Modelling and Simulation"

[6] William Graham, "Computer simulation and non linear dynamics"

[7] W.David Kelton ,"Simulation with Arena"
[8] Sankar Sengupta, "System simulation and modelling"

[9] Frank Hoppen,"Analysis and simulation of chaotic systems"

[10]P.Senthilkumar,"System simulation modeling and languages" 